\title{
Fusarium virguliforme, a soybean sudden death syndrome fungus in Malaysian soil
}

\author{
Khosrow Chehri • Baharuddin Salleh • Latiffah Zakaria
}

Received: 19 October 2013 / Accepted: 12 March 2014 / Published online: 20 March 2014

(C) Australasian Plant Pathology Society Inc. 2014

\begin{abstract}
Numerous Fusarium species associated with soil and different plants have been reported from Malaysia. Until now there are no reports on $F$. virguliforme in Malaysia. Fusarium virguliforme is the etiological agent of soybean sudden death syndrome (SDS). Morphological studies combined with molecular analysis using sequences from the translation elongation factor $1 \alpha(\mathrm{TEF}-1 \alpha)$ gene and nuclear ribosomal DNA internal transcribed spacer (ITS) regions were conducted to identify $F$. virguliforme $(2.1 \%)$ isolates from soil in Malaysia.
\end{abstract}

Keywords Fusarium solani species complex · Malaysia · ITS regions $\cdot$ TEF- $1 \alpha$

Fusarium solani species complex (FSSC) Clade 2 encompasses at least eight phylogenetic species including $F$. phaseoli (bean root rot pathogen), Fusarium virguliforme and related species that have been reported from all major plantation regions in South America (Aoki et al. 2003). Sudden death syndrome of soybean, caused by Fusarium virguliforme, is one of the most destructive diseases affecting soybean production in North and South America (Aoki et al. 2003). Aoki et al. (2003) extensively investigated the bean root rot pathogen and SDS pathogenic strains from North and South America based on comprehensive morphological comparisons and molecular phylogenetic analyses of multilocus DNA sequences Table 1. According to this study, they described three morphologically and phylogenetically distinct species within Clade 2. Fusarium virguliforme (formerly known as F. solani f. sp. glycine) and F. phaseoli (formerly known as F. solani $\mathrm{f}$.

K. Chehri $(\bowtie) \cdot$ B. Salleh $\cdot$ L. Zakaria

School of Biological Sciences, Universiti Sains Malaysia,

11800 Penang, Malaysia

e-mail: khchehri@gmail.com sp. phaseoli) were described for the United States strains and F. tucumaniae was illustrated for Argentinian strains (Roy 1997; Rupe et al. 2001). Aoki et al. (2005, 2012), based on detailed morphological comparisons, phenotypic and molecular phylogenetic analyses of multiple loci of DNA sequences, described $F$. brasiliense, F. cuneirostrum and F. crassistipitatum as novel SDS pathogens. Until now, no attempt has been made to classify members of the FSSC in Malaysia. Therefore, the objectives of the present study were to re-identify strains of FSSC stored in the Fusarium Culture Collection Unit of School of Biological Sciences, Universiti Sains Malaysia by using morphological and molecular markers. In this survey, 140 strains were investigated based on morphological characteristics as shown in Table 2. All strains were purified by the singlespore isolation technique. Single germinated macroconidia were transferred onto PDA plates and colony appearance was used to select isolates for further study. Fusarium strains were grown on PDA and carnation leaf agar (CLA) (Fisher et al. 1982) in $9 \mathrm{~cm}$ plastic Petri dishes. Cultures were incubated under $12 \mathrm{~h}$ alternating light (black/white) at $25 \pm 2{ }^{\circ} \mathrm{C}$ for 1 week. Colony morphology and colour were based primarily on cultures grown on PDA. Cited colours are given according to Kornerup and Wanscher (1978). For comparison of mycelial growth rates, agar blocks ca $5 \times 5 \mathrm{~mm}$ were cut from the margins of 1 week old cultures on CLA and transferred onto PDA and incubated at $25^{\circ} \mathrm{C}$ for 1 week in the dark. Thirty randomly selected conidia of each septation class (macroconidia and microconidia), sporodochial phialides, chlamydospores and conidiophores were measured and analysed by the 2-Sample T-Test using MINITAB ${ }^{\circledR} 15$ (Table 2). For species determination, the descriptions by Aoki et al. (2003, 2005, 2012) were adopted.

Representative FSSC strains were grown on PDA with sterile dialysis membranes (Lui et al. 2000) for 5 days (Table 3). The mycelium was harvested and ground in a sterile mortar with liquid nitrogen to a fine powder and then DNA 
Table 1 Strains of Fusarium solani species complex recovered from various hosts and substrates in Malaysia

\begin{tabular}{|c|c|c|c|c|}
\hline Strain number & Species & Origin & Host & Substrate and symptoms \\
\hline${ }^{\mathrm{a}} \mathrm{USM}{ }^{\mathrm{b}} \mathrm{FSSC}-{ }^{\mathrm{c}} \mathrm{P} 3 \mathrm{P}$ & F. solani & Penang & Potato & Tuber, dry rot \\
\hline USM FSSC-P53B & F. solani & Penang & Bean sprout & Root, rot \\
\hline USM FSSC-P54B & F. solani & Penang & Bean sprout & Root, rot \\
\hline USM FSSC-P57G & F. solani & Penang & Onion & Leaf, rot \\
\hline USM FSSC-P43R & F. solani & Penang & Rice & Seed \\
\hline USM FSSC-P0112S & F. solani & Penang & Soil & - \\
\hline USM FSSC-P104S & F. solani & Penang & Soil & - \\
\hline USM FSSC-P1235A & F. solani & Penang & Asparagus & Stem, brown spot \\
\hline USM FSSC-P21H & F. solani & Penang & Human & Finger \\
\hline USM FSSC-P200S & F. solani & Penang & Soil & - \\
\hline USM FSSC-P1361G & F. solani & Penang & Onion & Bulb, rot \\
\hline USM FSSC-P1531S & F. solani & Penang & Soil & - \\
\hline USM FSSC-P1532S & F. solani & Penang & Soil & - \\
\hline USM FSSC-P1555S & F. solani & Penang & Soil & - \\
\hline USM FSSC-P1557S & F. solani & Penang & Soil & - \\
\hline USM FSSC-P2103S & F. solani & Penang & Sand & - \\
\hline USM FSSC-P1194W & F. solani & Penang & Watermelon & $\begin{array}{l}\text { Stem, blackish brown } \\
\text { leaves }\end{array}$ \\
\hline USM FSSC-P1225W & F. solani & Penang & Watermelon & $\begin{array}{l}\text { Stem, blackish brown } \\
\text { leaves }\end{array}$ \\
\hline USM FSSC-P767M & F. solani & Penang & Mango & Stem \\
\hline USM FSSC-P2214An & F. solani & Penang & Angsana & - \\
\hline USM FSSC-P2142An & F. solani & Penang & Angsana & - \\
\hline USM FSSC-P2143An & F. solani & Penang & Angsana & - \\
\hline USM FSSC-P5004B & F. solani & Penang & Long bean & Leaf, spot \\
\hline USM FSSC-P1517S & F. solani & Penang & Soil & - \\
\hline USM FSSC-P1531S & F. solani & Penang & Soil & - \\
\hline USM FSSC-P3602Gr & F. solani & Penang & Digitaria setigera & Root, rot \\
\hline USM FSSC-P3598Gr & F. solani & Penang & Paspalum orbiculare & Root, rot \\
\hline USM FSSC-P52B & F. solani & Penang & Bean sprout & Root, rot \\
\hline USM FSSC-P993P & F. solani & Penang & Potato & Tuber, dry rot \\
\hline USM FSSC-P1263S & F. solani & Penang & Soil & - \\
\hline USM FSSC-P878V & F. solani & Penang & Vanda & Black, stem rot \\
\hline USM FSSC-P6S & F. solani & Penang & Soil & - \\
\hline USM FSSC-P2108S & F. solani & Penang & Soil & - \\
\hline USM FSSC-P17E & F. solani & Penang & Eggplant & Stem, rot \\
\hline USM FSSC-P2106S & F. solani & Penang & Soil & - \\
\hline USM FSSC-P65S & F. solani & Penang & Soil & - \\
\hline USM FSSC-P2109S & F. solani & Penang & Soil & - \\
\hline USM FSSC-P76V & F. solani & Penang & Cassava & Leaf, spot \\
\hline USM FSSC-P0111S & F. solani & Penang & Soil & - \\
\hline USM FSSC-P86S & F. solani & Penang & Soil & - \\
\hline USM FSSC-P88S & F. solani & Penang & Soil & - \\
\hline USM FSSC-P1980S & F. solani & Penang & Soil & - \\
\hline USM FSSC-P91S & F. solani & Penang & Soil & - \\
\hline USM FSSC-P93S & F. solani & Penang & Soil & - \\
\hline USM FSSC-P97S & F. solani & Penang & Soil & - \\
\hline USM FSSC-P98S & F. solani & Penang & Soil & - \\
\hline USM FSSC-P34L & F. solani & Penang & Oil palm & Seed \\
\hline
\end{tabular}


Table 1 (continued)

\begin{tabular}{|c|c|c|c|c|}
\hline Strain number & Species & Origin & Host & Substrate and symptoms \\
\hline USM FSSC-P156R & F. solani & Penang & Rice & Seed \\
\hline USM FSSC-P178R & F. solani & Penang & Rice & Seed \\
\hline USM FSSC-P183S & F. solani & Penang & Soil & - \\
\hline USM FSSC-P1282S & F. solani & Penang & Soil & - \\
\hline USM FSSC-P383N & F. solani & Penang & Banana & Fruit, rot \\
\hline USM FSSC-P391N & F. solani & Penang & Banana & Fruit, rot \\
\hline USM FSSC-P458N & F. solani & Penang & Banana & Fruit, rot \\
\hline USM FSSC-B565S & F. solani & Selangor & Soil & - \\
\hline USM FSSC-B636S & F. solani & Selangor & Soil & - \\
\hline USM FSSC-B1409M & F. solani & Selangor & Mango & Fruit, spot \\
\hline USM FSSC-B1479G & F. solani & Selangor & Ginger & Root, vascular wilt \\
\hline USM FSSC-B1481G & F. solani & Selangor & Ginger & Root, vascular wilt \\
\hline USM FSSC-B1765S & F. solani & Selangor & Soil & - \\
\hline USM FSSC-B1766S & F. solani & Selangor & Soil & - \\
\hline USM FSSC-B1767S & F. solani & Selangor & Soil & - \\
\hline USM FSSC-B1769S & F. solani & Selangor & Soil & - \\
\hline USM FSSC-B1770S & F. solani & Selangor & Soil & - \\
\hline USM FSSC-B2285L & F. solani & Selangor & Oil palm & Crown, rot \\
\hline USM FSSC-B2466N & F. solani & Selangor & Banana & Root, vascular wilt \\
\hline USM FSSC-B2982\$ & F. solani & Selangor & Coffee & Stem, dieback \\
\hline USM FSSC-B2983\$ & F. solani & Selangor & Coffee & Stem, dieback \\
\hline USM FSSC-B2989Br & F. solani & Selangor & Brinjals & Stem \\
\hline USM FSSC-B3401An & F. solani & Selangor & Angsana & - \\
\hline USM FSSC-B3819Gr & F. solani & Selangor & Ischaemum magnum & Leaf, rot \\
\hline USM FSSC-B3823Gr & F. solani & Selangor & Eragrostis amabilis & Stem, rot \\
\hline USM FSSC-B3824Gr & F. solani & Selangor & Eragrostis amabilis & Leaf, rot \\
\hline USM FSSC-B3827Gr & F. solani & Selangor & Dactyloctenium aegyptium & Root \\
\hline USM FSSC-B3829Gr & F. solani & Selangor & Dactyloctenium aegyptium & Root \\
\hline USM FSSC-B3830Gr & F. solani & Selangor & Dactyloctenium aegyptium & Root \\
\hline USM FSSC-B3832Gr & F. solani & Selangor & Digitaria ciliaris & Root \\
\hline USM FSSC-B3834Gr & F. solani & Selangor & Pennisetum purpureum & Root \\
\hline USM FSSC-B3846Gr & F. solani & Selangor & Echinochloa colona & Root \\
\hline USM FSSC-B3822Gr & F. solani & Selangor & Eragrostis amabilis & Root \\
\hline USM FSSC-B3024 & F. solani & Selangor & UPM 31-P1 & - \\
\hline USM FSSC-A3034L & F. solani & Perak & Oil palm & Crown, rot \\
\hline USM FSSC-A1966S & F. solani & Perak & Soil & - \\
\hline USM FSSC-A491S & F. solani & Perak & Soil & - \\
\hline USM FSSC-A998W & F. solani & Perak & Watermelon & Root, rot \\
\hline USM FSSC-A1444S & F. solani & Perak & Soil & - \\
\hline USM FSSC-A1449S & F. solani & Perak & Banana & Root, vascular wilt \\
\hline USM FSSC-A1450N & F. solani & Perak & Banana & Root, vascular wilt \\
\hline USM FSSC-A1463N & F. solani & Perak & Banana & Root, vascular wilt \\
\hline USM FSSC-A1863W & F. solani & Perak & Watermelon & Seed \\
\hline USM FSSC-A1881W & F. solani & Perak & Watermelon & Fruit, vascular wilt \\
\hline USM FSSC-A1882W & F. solani & Perak & Watermelon & Fruit, vascular wilt \\
\hline USM FSSC-A1883W & F. solani & Perak & Watermelon & Stem, vascular wilt \\
\hline USM FSSC-A2333Gr & F. solani & Perak & Jojoba & Root, wilt \\
\hline USM FSSC-A263R & F. solani & Perak & Roselle & Root, rot \\
\hline USM FSSC-A1901S & F. solani & Perak & Soil & - \\
\hline
\end{tabular}


Table 1 (continued)

\begin{tabular}{|c|c|c|c|c|}
\hline Strain number & Species & Origin & Host & Substrate and symptoms \\
\hline USM FSSC-A1909W & F. solani & Perak & Watermelon & Root, lesion \\
\hline USM FSSC-A1910W & F. solani & Perak & Watermelon & Root, lesion \\
\hline USM FSSC-A1936W & F. solani & Perak & Watermelon & Soil \\
\hline USM FSSC-A1937W & F. solani & Perak & Watermelon & Stem, vascular wilt \\
\hline USM FSSC-A1968W & F. solani & Perak & Watermelon & Root \\
\hline USM FSSC-A1969W & F. solani & Perak & Watermelon & Root \\
\hline USM FSSC-A1970W & F. solani & Perak & Watermelon & Stem, vascular wilt \\
\hline USM FSSC-A1973W & F. solani & Perak & Watermelon & Root \\
\hline USM FSSC-A1974W & F. solani & Perak & Watermelon & Root \\
\hline USM FSSC-A1977W & F. solani & Perak & Watermelon & Stem, vascular wilt \\
\hline USM FSSC-A2072W & F. solani & Perak & Watermelon & Root \\
\hline USM FSSC-A2073S & F. solani & Perak & Watermelon & Soil, vascular wilt \\
\hline USM FSSC-Q724Q & F. solani & Sarawak & Sorghum & Stalk, rot \\
\hline USM FSSC-Q725Co & F. solani & Sarawak & Cocoa & - \\
\hline USM FSSC-Q726D & F. solani & Sarawak & Black pepper & - \\
\hline USM FSSC-Q4992D & F. solani & Sarawak & - & Flower \\
\hline USM FSSC-Q728 & F. solani & Sarawak & - & Flower \\
\hline USM FSSC-Q729D & F. solani & Sarawak & Black pepper & - \\
\hline USM FSSC-Q737 & F. solani & Sarawak & - & Flower \\
\hline USM FSSC-Q1014Q & F. solani & Sarawak & Sorghum & Fruit \\
\hline USM FSSC-Q1015Q & F. solani & Sarawak & Sorghum & Fruit \\
\hline USM FSSC-Q1016Q & F. solani & Sarawak & Sorghum & Fruit \\
\hline USM FSSC-Q1017Q & F. solani & Sarawak & Sorghum & Root \\
\hline USM FSSC-Q1018Q & F. solani & Sarawak & Sorghum & Root \\
\hline USM FSSC-Q1021Q & F. solani & Sarawak & Sorghum & Root \\
\hline USM FSSC-Q1022Q & F. solani & Sarawak & Sorghum & Root \\
\hline USM FSSC-Q1023Q & F. solani & Sarawak & Sorghum & Root \\
\hline USM FSSC-Q1024Q & F. solani & Sarawak & Sorghum & Root \\
\hline USM FSSC-Q1025Q & F. solani & Sarawak & Sorghum & Root \\
\hline USM FSSC-Q1026Q & F. solani & Sarawak & Sorghum & Root \\
\hline USM FSSC-Q1027Q & F. solani & Sarawak & Sorghum & Root \\
\hline USM FSSC-Q1033Q & F. solani & Sarawak & Sorghum & Root \\
\hline USM FSSC-Q1034Q & F. solani & Sarawak & Sorghum & Root \\
\hline USM FSSC-Q1035Q & F. solani & Sarawak & Sorghum & Fruit \\
\hline USM FSSC-Q1036Q & F. solani & Sarawak & Sorghum & Root \\
\hline USM FSSC-Q1037Q & F. solani & Sarawak & Sorghum & Fruit \\
\hline USM FSSC-Q1038Q & F. solani & Sarawak & Sorghum & Fruit \\
\hline USM FSSC-Q1039Q & F. solani & Sarawak & Sorghum & Fruit \\
\hline USM FSSC-Q1165Gr & F. solani & Sarawak & Grass & - \\
\hline USM FSSC-Q1162Fe & F. solani & Sarawak & Feces & Feces \\
\hline USM FSSC-D992S & F. virguliforme & Kelantan & Soil & - \\
\hline USM FSSC-T531S & F. virguliforme & Terengganu & Soil & - \\
\hline USM FSSC-J969S & F. virguliforme & Johor & Soil & - \\
\hline
\end{tabular}

${ }^{\text {a } U S M ~ U n i v e r s i t i ~ S a i n s ~ M a l a y s i a ~}$

${ }^{\mathrm{b}}$ FSSC Fusarium solani species complex

${ }^{\mathrm{c}}$ P3P The first letter denotes a particular state in Malaysia (A- Perak, B- Selangor, D- Kelantan, J- Johor, P- Penang, Q- Sarawak, and S- Sabah) and the P symbol represents the host i.e. potato 
Table 2 Comparison of morphological characteristics of representative strains within the Fusarium solani species complex

\begin{tabular}{|c|c|c|c|c|c|}
\hline \multirow[t]{2}{*}{ Strain number } & \multirow[t]{2}{*}{ Species } & \multirow[t]{2}{*}{ Shape of microconidia } & \multirow{2}{*}{$\begin{array}{l}\text { Shape of basal cell } \\
\text { and apical cell }\end{array}$} & \multicolumn{2}{|c|}{ Length $\times$ width of macroconidia $(\mu \mathrm{m})^{\mathrm{a}}$} \\
\hline & & & & 3-septate & 5-septate \\
\hline USM FSSC-B1409M & F. solani & Oval, clavate, reniform & $\begin{array}{l}\text { Barely notched and } \\
\text { papillate curved }\end{array}$ & $41.5 \pm 1.5 \times 5.4 \pm 0.2$ & $45.5 \pm 2.5 \times 5.8 \pm 0.2$ \\
\hline USM FSSC-Q1371W & F. solani & Oval, clavate, reniform & $\begin{array}{l}\text { Barely notched and } \\
\text { papillate curved }\end{array}$ & $41.5 \pm 1.5 \times 5.5 \pm 0.2$ & $45.5 \pm 2.5 \times 5.9 \pm 0.2$ \\
\hline USM FSSC-Q1165Gr & F. solani & Oval, clavate, reniform & $\begin{array}{l}\text { Barely notched and } \\
\text { papillate curved }\end{array}$ & $40.5 \pm 1.5 \times 5.4 \pm 0.2$ & $45.5 \pm 2.5 \times 5.8 \pm 0.2$ \\
\hline USM FSSC-T531S & F. virguliforme & $\begin{array}{l}\text { Comma-shaped, } \\
\text { elongated oval }\end{array}$ & $\begin{array}{l}\text { Distinctly notched } \\
\text { and tapered, } \\
\text { curved }\end{array}$ & $54.5 \pm 2.5 \times 5.6 \pm 0.5$ & $59.5 \pm 2.5 \times 5.7 \pm 0.5$ \\
\hline USM FSSC-J969S & F. virguliforme & $\begin{array}{l}\text { Comma-shaped, } \\
\text { elongated oval }\end{array}$ & $\begin{array}{l}\text { Distinctly notched } \\
\text { and tapered, curved }\end{array}$ & $54.5 \pm 2.5 \times 5.8 \pm 0.5$ & $57.5 \pm 2.5 \times 5.6 \pm 0.5$ \\
\hline USM FSSC-D992S & F. virguliforme & $\begin{array}{l}\text { Comma-shaped, } \\
\text { elongated oval }\end{array}$ & $\begin{array}{l}\text { Distinctly notched } \\
\text { and tapered, } \\
\text { curved }\end{array}$ & $55.5 \pm 1.5 \times 5.8 \pm 0.5$ & $61.0 \pm 2.5 \times 5.8 \pm 0.5$ \\
\hline
\end{tabular}

${ }^{a}$ Mean values of 30 random conidia \pm standard deviation

was extracted using a DNeasy ${ }^{\circledR}$ Plant Mini Kit (Qiagen) according to the manufacturer's instruction. Amplification of the translation elongation factor- $1 \alpha$ (tefl) gene, and internal transcribed spacer (ITS) regions was conducted utilising the primer pair efl and ef2 for tefl (O'Donnell et al. 1998), and ITS1 and ITS4 for ITS region (White et al. 1990). PCRs were performed in a Peltier Thermal Cycler, PTC-100 ${ }^{\circledR}$ (MJ Research, Inc. USA) in a total volume of $25 \mu$. The PCR mixture contained $4 \mu \mathrm{l} 5 \times$ buffer (Promega, Madison, WI, USA), $4 \mathrm{mM} \mathrm{MgCl}, 0.2 \mathrm{mM}$ deoxynucleotide triphosphate (dNTP) (Promega), $0.8 \mu \mathrm{M}$ of each primer, 0.75 units of Taq DNA polymerase (Promega ${ }^{\circledR}$, USA), and 6 ng of template DNA. To prevent evaporation, the reactions were overlaid with $25 \mu \mathrm{l}$ of sterilised mineral oil. PCR products were purified using Qiagen columns according to the manufacturer's instructions and stored at $-20{ }^{\circ} \mathrm{C}$. The purified PCR products were sent to First BASE Laboratories Sdn. Bhd. for sequencing of tefl gene and ITS regions in both directions (forward and reverse) using $\mathrm{ABI}$ 3730x1 model of sequencer. Forward and reversed sequences of tefl gene and ITS regions were edited and aligned using BioEdit version 7.0.5 (Hall 1999). Consensus sequences were used as query to search for similarities using two sources: I. BLAST network services at the National Centre for Biotechnology Information (NCBI); II. FUSARIUM-ID v.1.0 database (http://fusarium.cbio. psu.edu) (Geiser et al. 2004).

All FSSC strains were successfully investigated based on macroscopic and microscopic characteristics. One-hundred and thirty seven strains $(97.9 \%)$ were identified as $F$. solani and three strains (2.1\%) belonged to F. virguliforme (Fig. 1). All three strains produced pink to bluish-gray mycelium. Aerial conidiophores, unbranched or sparsely branched, up to $250 \mu \mathrm{m}$ long and $2.5-7.0 \mu \mathrm{m}$ wide at base, abundantly formed on CLA at $25{ }^{\circ} \mathrm{C}$ (Fig. 1a). Aerial phialides were simple, cylindrical or subcylindrical. Sporodochial conidiophores were subcylindrical or ampulliform with distinctive collarette at the tip, 20-28 $\mu \mathrm{m}$ long, 2.5-3.0 $\mu \mathrm{m}$ wide at base (Fig. 1b). Macroconidia arising from sporodochia were falcate and sometimes widest at middle, with 3-5-septa and mostly 3septate, with a tapered and curved apical cell and distinctly notched basal cell (Fig. 1c-d). Microconidia were comma-

Table 3 Strain number, geographical origin, and GenBank accession numbers of representative strains within the Fusarium solani species complex

\begin{tabular}{|c|c|c|c|c|c|}
\hline Strain number & Species & ${ }^{\mathrm{a}}$ tef1 & ${ }^{\mathrm{a}} \mathrm{ITS}$ & Substrate & Origin \\
\hline USM FSSC-D992S & F.virguliforme & JX970965 & JX982562 & Soil & Kelantan \\
\hline USM FSSC-T531S & F.virguliforme & JX970966 & JX982563 & Soil & Banana field, Terengganu \\
\hline USM FSSC-J969S & F.virguliforme & JX970967 & JX982564 & Soil & Banana field, Johor \\
\hline USM FSSC-B1409M & F.solani & KF836690 & KF836675 & Mango & Selangor \\
\hline USM FSSC-Q1371W & F.solani & KF836691 & KF836676 & Watermelon & Sarawak \\
\hline USM FSSC-Q1165Gr & F.solani & KF836693 & KF836678 & Grass & Sarawak \\
\hline
\end{tabular}

${ }^{\mathrm{a}}$ GenBank numbers for translation elongation factor 1-alpha (tefl) partial sequences, and the ITS rDNA region 
shaped, elongated oval to sometimes short-clavate and ellipsoidal shaped with a swollen apex often rounded and mostly 0 -2-septate (Fig. 1f). The size of conidia; 0-1(-2)-septate = (11-) 15.6-26 (-33.1) $\times(3.8-)$ 5-6.4 (-7.4) $\mu \mathrm{m}$; 3-septate $=$ (30-) 47-56 (-65) × (4.2-) 4.6-5.4 (-6) $\mu \mathrm{m} ; 4$-septate $(45-)$ $53-58(-65) \times(4.7-) 5.2-5.4(-6) \mu \mathrm{m} ; 5$-septate $56-65 \times 5.3-$ $6.1 \mu \mathrm{m}$. Two types of chlamydospores formed, relatively abundant in mycelium, smooth and rough-walled, 5-14 $\mu \mathrm{m}$ in diameter, may occasionally be found within macroconidia. Chlamydospores formed mostly singly, and in pairs, rarely in chains, mostly subglobose, terminal or intercalar (Fig. 1e).

A list of species names and culture collection numbers, geographical origins, original substrates, and GenBank accession numbers of the individual strains used in this study are in Table 3. Single bands of DNA fragments approximately 550-bp and 700-bp were successfully amplified for ITS region and tefl gene from all three strains, respectively. From similarities searched at NCBI and FUSARIUM-ID database, all three strains were similar to $F$. virguliforme with the percentage of maximum identity (100\%). Based on morphological features combined with molecular analysis using tefl gene and ITS regions sequences, $F$. virguliforme was reported for the first time in Malaysia.

Fusarium virguliforme is one of the most important causal agents of sudden death syndrome (SDS) in soybean and has been reported from all major growing regions in North
Fig. 1 Fusarium virguliforme grown on CLA, 2 weeks, $25^{\circ} \mathrm{C}$, cultured in the dark. a Slender, unbranched aerial conidiophores. b Branched sporodochial conidiophores forming falcate to curved cylindrical conidia. $\mathbf{c}-\mathbf{d}$ Falcate and comma-shaped macroconidia produced in sporodochial. e Terminal and intercalary chlamydospores, and produced chlamydospores in conidia. f Comma-shaped microconidia were formed on conidiophores in hyphae. Scale bars: $\mathbf{a}=50 \mu \mathrm{m}, \mathbf{b}-\mathbf{f}=20 \mu \mathrm{m}$
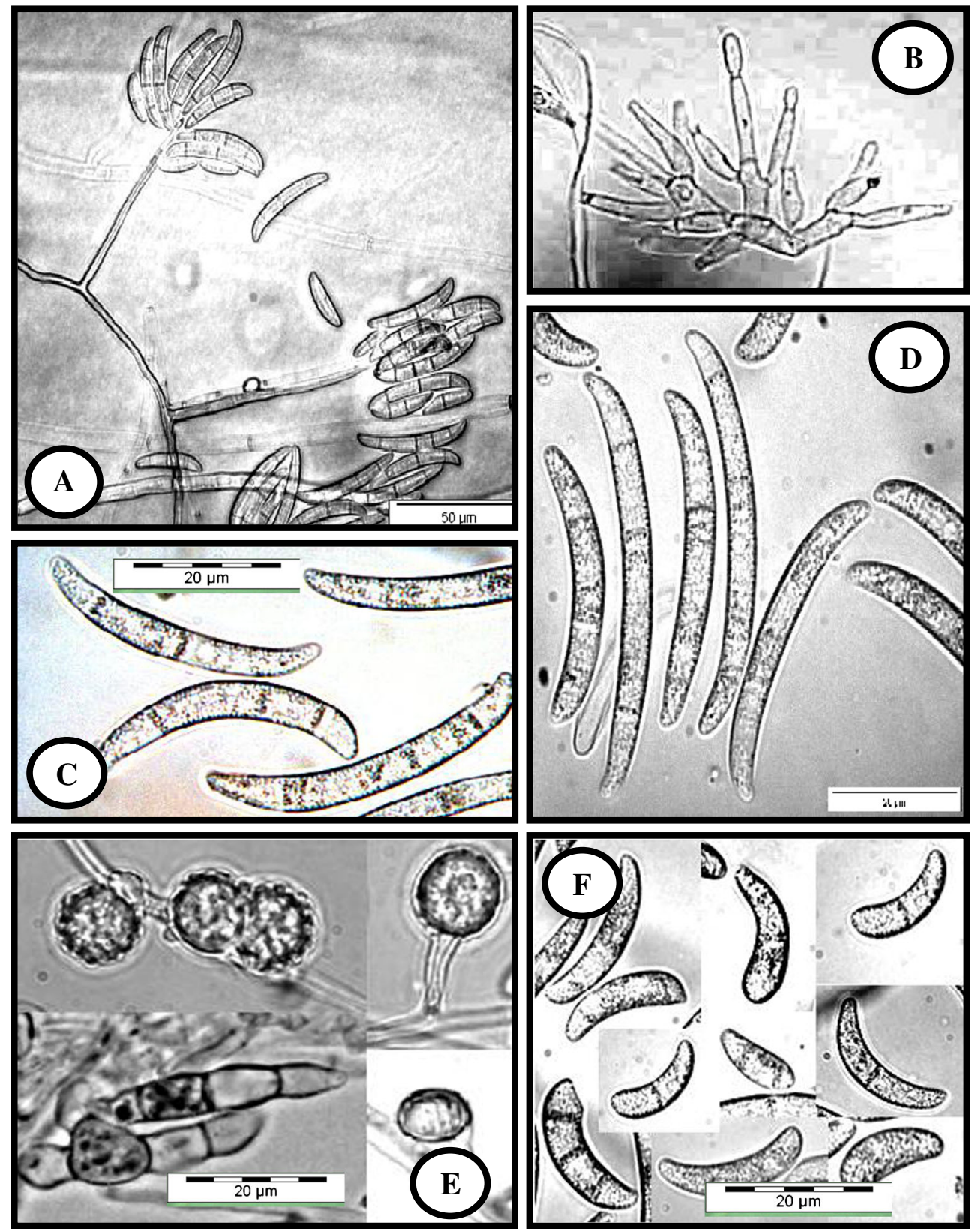
America (Aoki et al. 2003, 2005, 2012). Kolander et al. (2012) showed that the diversity and number of hosts for $F$. virguliforme are greater than previously reported by Aoki et al. $(2005,2012)$ and indicated that agricultural crops other than soybean can be damaged by $F$. virguliforme and increase pathogen inoculum in soil. Therefore, further information regarding this well-known plant pathogen within the region is needed. To the best of our knowledge this is the first report on occurrence of this species in agricultural soil in Malaysia.

\section{References}

Aoki T, O'Donnell K, Homma Y, Lattanzi A (2003) Sudden-death syndrome of soybean is caused by two morphologically and phylogenetically distinct species within the Fusarium solani species complex-F. virguliforme in North America and F. tucumaniae in South America. Mycologia 95:660-684

Aoki T, O'Donnell K, Scandiani MM (2005) Sudden death syndrome of soybean in South America is caused by four species of Fusarium: Fusarium brasiliense sp. nov., F. cuneirostrum sp. nov., F. tucumaniae, and F. virguliforme. Mycoscience 46:162-183

Aoki T, Scandiani MM, O'Donnell K (2012) Phenotypic, molecular phylogenetic, and pathogenetic characterization of Fusarium crassistipitatum sp. nov., a novel soybean sudden death syndrome pathogen from Argentina and Brazil. Mycoscience 53:167-186

Fisher NL, Burgess LW, Toussoun TA, Nelson PE (1982) Carnation leaves as a substrate and for preserving cultures of Fusarium species. Phytopathology 72:151-153
Geiser DM, Jimenz Gasco MM, Kang S, Mkalowska I, Veeraraghavan N, Ward TJ, Zhang N, Kuldau GA, O’Donnell K (2004) FUSARIUMIDv.1.0: A DNA sequence database for identifying Fusarium. Eur J Plant Pathol 110:473-479

Hall TA (1999) BioEdit: a user-friendly biological sequence alignment editor and analysis program for Windows 95/98/NT. Nucleic Acids Symp Ser 41:95-98

Kolander TM, Bienapfl JC, Kurle JE, Malvick DK (2012) Symptomatic and asymptomatic host range of Fusarium virguliforme, the causal agent of soybean sudden death syndrome. Plant Dis 96:1148-1156

Kornerup A, Wanscher JH (1978) Methuen handbook of colour, 3rd edn. Eyre Methuen, London, $252 \mathrm{p}$

Lui D, Coloe S, Baird R, Pedersen J (2000) Rapid mini-preparation of fungal DNA for PCR. J Clin Microbiol 38:471-477

O’Donnell K, Kistler HC, Cigelnike E, Ploetz RC (1998) Multiple evolutionary origins of the fungus causing Panama disease of banana: Concordant evidence from nuclear and mitochondrial gene genealogies. Proc Natl Acad Sci U S A 95:2044-2049

Roy KW (1997) Fusarium solani on soybean roots: nomenclature of the causal agent of sudden-death syndrome and identity and relevance of $F$. solani form B. Plant Dis 81:259-266

Rupe JC, Correll JC, Guerber JC, Becton CM, Gbur EE, Cummings MS, Yount PA (2001) Differentiation of the sudden death syndrome pathogen of soybean, Fusarium solani f. sp. glycines, from other isolates of $F$. solani based on cultural morphology, pathogenicity, and mitochondrial DNA restriction fragment length polymorphisms. Can J Bot 79:829-835

White TJ, Bruns T, Lee S, Taylor J (1990) Amplification and direct sequencing of fungal ribosomal RNA genes for phylogenetics. In: Innis MA, Gelfand DH, Sninsky JJ, White TJ (eds) PCR protocols: a guide to methods and applications. Academic, San Diego, pp 315322 